Noname manuscript No.

(will be inserted by the editor)

\title{
B-Lift Curves in Euclidean 3-space
}

\author{
Anıl Altınkaya and Mustafa Çalışkan
}

Received: date / Accepted: date

\begin{abstract}
In this study, we introduce a new type curve in 3-dimensional space which called B-lift curve and we obtain the Frenet operators of the B-lift curve. Moreover, we consider the correpondence of Frenet operators between the Blift curve and the natural lift curve. Finally, we investigate the B-lift curve according to the main curve is slant helix or darboux helix.
\end{abstract}

Mathematics Subject Classification (2010) 53A04, 53A15

Keywords B-Lift, Natural Lift, Slant helix, Darboux helix

\section{Introduction}

The theory of curves plays an important role in differential geometry, kinematics, computer design, etc. One of the well-known curves is the natural lift curve. The definition of the natural lift was first encountered in Thorpe's book, see [1]. According to the definition, the natural lift curve was formed by combining the end points of unit tangent vectors of the main curve. Natural lift curves have attracted the attention of many mathematicians. M. Çalıskan and E. Ergün [4] introduced Frenet operators of the natural lift curve in terms of the Frenet operators of the main curve.

A regular curve $\gamma$ in $\mathbb{R}^{3}$ is characterized by the situation of the curvature and the torsion. For example, a straight line has no curvature (i.e. $\kappa=0$ ) and a plane curve has no torsion (i.e. $\tau=0$ ). [2] A curve $\gamma$ is called general helix

A. Altinkaya

Department of Mathematics, Faculty of Science, Gazi University, Emniyet Mahallesi, Bandırma Cd.No:6/9, 06200 Yenimahalle, Ankara, Turkey

E-mail: anilaltinkaya@gazi.edu.tr

M. Çalışkan

Department of Mathematics, Faculty of Science, Gazi University, Emniyet Mahallesi, Bandırma Cd.No:6/9, 06200 Yenimahalle, Ankara, Turkey

E-mail: mustafacaliskan@gazi.edu.tr 
in Euclidean 3-space if tangent vector of the curve makes a constant angle with a fixed straight line. If both $\kappa \neq 0$ and $\tau>0$ are constant, a curve $\gamma$ is called circular helix. [2] In 1802, M. A. Lancert proved that the ratio of the curvatures is constant along the curve in general helix (or cylindirical helix).

Many authors have written articles on helices and different types of helices. S. Izumiya and N. Takeuchi [9] defined slant helices as the principal normal vector makes a constant angle with a fixed direction. They have also introduced conical geodesic curve. L. Kula, N. Ekmekci, Y. Yaylı, K. İlarslan [11] gave some characterizations of slant helices and introduced Frenet operators of slant helices in $\mathbb{R}^{3}$. E. Zıplar, A. Şenol and Y. Yaylı [12] defined Darboux helix that Darboux vector makes a constant angle with a fixed straight line. Morever, they gave the relation between slant helices and darboux helices.

However, there is no research about B-lift curves. In this paper, based on Thorpe's definition, B-Lift curve is defined and some characterizations of the Frenet operators are given. We also investigate the relation between the Frenet operators of the B-lift curve and the Frenet operators of natural lift curve. Furthermore, we examine the status of the B-Lift curve according to whether the main curve is slant helix or darboux helix. Finally, some examples are given and we draw our curves with Mathematica program.

\section{Preliminaries}

In this section, some basic definitions and theorems in differential geometry are given. Furthermore, some properties of the natural lift curve, general helix, slant helix and Darboux helix are denoted.

Let $\vec{A}=\left(a_{1}, a_{2}, a_{3}\right)$ be a vector in $\mathbb{R}^{3}$. The norm is defined as $\|\vec{A}\|=$ $\sqrt{a_{1}^{2}+a_{2}^{2}+a_{3}^{2}}$. If $\|\vec{A}\|$ is equal to 1 , then $\vec{A}$ is called unit vector in $\mathbb{R}^{3}$. For the vectors $\vec{A}=\left(a_{1}, a_{2}, a_{3}\right)$ and $\vec{B}=\left(b_{1}, b_{2}, b_{3}\right)$ in $\mathbb{R}^{3}$, the inner product is defined as $\langle\vec{A}, \vec{B}\rangle=a_{1} b_{1}+a_{2} b_{2}+a_{3} b_{3}$.

A parametrized curve $\gamma: I \rightarrow \mathbb{R}^{3}$ is called regular curve if $\gamma^{\prime}(s) \neq 0$, where $\mathrm{s} \in \mathrm{I}$. Let $\gamma$ be a curve in $\mathbb{R}^{3}$, if $\gamma^{\prime}(s)$ is equal to 1 then the curve $\gamma: \mathrm{I} \rightarrow \mathbb{R}^{3}$ is a unit speed curve.

Let $\gamma$ be a unit speed curve in $\mathbb{R}^{3}$. T $(\mathrm{s}), \mathrm{N}(\mathrm{s}), \mathrm{B}(\mathrm{s})$ is called FrenetSerret frame of the curve $\gamma(s)$. T(s) $=\gamma^{\prime}(s)$ is the unit tangent vector of the curve $\gamma(s)$. The unit principal normal and binormal vectors defined by $N(s)=\frac{\gamma^{\prime \prime}(s)}{\left\|\gamma^{\prime \prime}(s)\right\|}$ and $B(s)=T(s) \times N(s)$, respectively. Frenet-Serret formulas of the unit speed curve $\gamma(s)$ are as follows:

$$
\begin{aligned}
T^{\prime}(s) & =\kappa(s) N(s), \\
N^{\prime}(s) & =-\kappa(s) T(s)+\tau(s) B(s), \\
B^{\prime}(s) & =-\tau(s) N(s),
\end{aligned}
$$

where $\kappa(s)=\left\|\gamma^{\prime \prime}(s)\right\|$ and $\tau(s)=-\left\langle B^{\prime}(s), N(s)\right\rangle$ are the curvature and torsion of the curve $\gamma(s)$, respectively [1]. 
Definition 1 Let $\gamma: \mathrm{I} \rightarrow \mathrm{M}$ be a unit speed curve, where $\mathrm{M} \subset \mathbb{R}^{3}$ be a hypersurface. We called an integral curve to the curve $\gamma$ if

$$
\gamma^{\prime}(s)=X(\gamma(s))
$$

where $\mathrm{X}$ is a differentiable vector field on $\mathrm{M}$ [4].

Definition 2 Let $\gamma: \mathrm{I} \rightarrow \mathrm{M}$ be a unit speed curve. The natural lift curve $\bar{\gamma}$ : $\mathrm{I} \rightarrow \mathrm{TM}$ of the curve $\gamma$ is defined as follows:

$$
\bar{\gamma}(s)=\left(\gamma(s), \gamma^{\prime}(s)\right)=\left.\gamma^{\prime}(s)\right|_{\gamma(s)} .
$$

Therefore, we can write

$$
\frac{d \bar{\gamma}(s)}{d s}=\left.\frac{d}{d s}\left(\gamma^{\prime}(s)\right)\right|_{\gamma(s)}=D_{\gamma^{\prime}(s)} \gamma^{\prime}(s)
$$

where D is Levi-Civita connection in $\mathbb{R}^{3}$ [4].

Theorem 1 Let $\bar{\gamma}(s)$ be the natural lift curve of $\gamma(s)$. Then there is the following equation between Frenet frames of the curves $\gamma(s)$ and $\bar{\gamma}(s)$ :

$$
\begin{aligned}
& \bar{T}(s)=N(s), \\
& \bar{N}(s)=-\frac{\kappa(s)}{\|W(s)\|} T(s)+\frac{\tau(s)}{\|W(s)\|} B(s), \\
& \bar{B}(s)=\frac{\tau(s)}{\|W(s)\|} T(s)+\frac{\kappa(s)}{\|W(s)\|} B(s),
\end{aligned}
$$

where $\{T(s), N(s), B(s)\}$ and $\{\bar{T}(s), \bar{N}(s), \bar{B}(s)\}$ are Frenet frames of the curve and its natural lift curve, respectively. The Darboux vector $W$ is presented as $W=\tau T+\kappa B$. Moreover, $\kappa=\|W\| \cos \varphi$ and $\tau=\|W\| \sin \varphi$ are represented as curvature ans torsion. Here, $\varphi$ is the angle between Darboux vector and binormal vector of $\gamma(s)$, see [4].

Theorem 2 Let $\bar{\gamma}(s)$ be the natural lift curve of $\gamma$ for a given regular curve $\gamma$. Then there are the following equations:

$$
\bar{\kappa}(s)=\frac{\kappa^{2}+\tau^{2}}{\|W\|}, \bar{\tau}(s)=\frac{-\kappa^{\prime}(s) \tau(s)+\kappa(s) \tau^{\prime}(s)}{\|W\|^{2}}
$$

where $\bar{\kappa}$ and $\bar{\tau}$ are the curvature and torsion of $\bar{\gamma}(s)$, respectively [4].

Proposition 1 Let $\gamma: I \rightarrow \mathbb{R}^{3}$ be a unit speed curve with curvature $\kappa$ and torsion $\tau$. The curve $\gamma$ is the general helix if and only if $\frac{\tau}{\kappa}$ is a constant [6].

Proposition 2 For any unit speed curve $\gamma: I \rightarrow \mathbb{R}^{3}$ with $\kappa \neq 0, \gamma$ is a slant helix if and only if

$$
\sigma(s)=\frac{\kappa^{2}}{\left(\kappa^{2}+\tau^{2}\right)^{\frac{3}{2}}}\left(\frac{\tau}{\kappa}\right)^{\prime}(s)
$$

is a constant fonction [9]. 
Theorem $3 \gamma: I \rightarrow \mathbb{R}^{3}$ is the Darboux helix if and only if

$$
\sigma^{*}(s)=\frac{\left(\kappa^{2}+\tau^{2}\right)^{\frac{3}{2}}}{\kappa^{2}} \frac{1}{\left(\frac{\tau}{\kappa}\right)^{\prime}(s)}
$$

is a constant function [12].

Theorem 4 Let $\gamma$ be a curve in $\mathbb{R}^{3}$. If $\frac{\kappa}{\tau}$ is not constant, then $\gamma$ is a slant helix if and only if $\gamma$ is a Darboux helix [12].

\section{B-Lift Curves in 3-Euclidean Space}

In this section, we describe the B-Lift curve which is the curve obtained by the end points of the binormal vectors of the main curve. We also obtain the Frenet operators of the B-lift curves and compare the Frenet operators of the B-Lift curves with the Frenet operators of the natural lift curves. Morever, we examine the case of the B-lift curve according to whether the main curve is slant helix or darboux helix and we give some examples with these situations.

Definition 3 For any unit speed curve $\gamma: \mathrm{I} \rightarrow \mathrm{M}, \gamma_{B}: \mathrm{I} \rightarrow \mathrm{TM}$ is called the B-Lift curve of $\gamma$ which provides the following equation:

$$
\gamma_{B}(s)=(\gamma(s), B(s))=\left.B(s)\right|_{\gamma(s)}
$$

where B is the binormal vector of the curve $\gamma$.

Theorem 5 Let $\gamma_{B}$ be the B-Lift curve of a regular curve $\gamma$. Then the following equations are provided:

$$
\begin{aligned}
T_{B}(s) & =-N(s), \\
N_{B}(s) & =\frac{\kappa(s)}{\|W(s)\|} T(s)-\frac{\tau(s)}{\|W(s)\|} B(s), \\
B_{B}(s) & =\frac{\tau(s)}{\|W(s)\|} T(s)+\frac{\kappa(s)}{\|W(s)\|} B(s)
\end{aligned}
$$

where $\{T(s), N(s), B(s)\}$ and $\left\{T_{B}(s), N_{B}(s), B_{B}(s)\right\}$ are the Frenet vectors of the curve $\gamma$ and its B-lift curve, respectively. Furthermore, $\kappa$ is the curvature, $\tau$ is the torsion and the Darboux vector $W=\tau T+\kappa B$ of the curve $\gamma$. (Specially torsion will be taken greater than zero.)

Proof. Let $\gamma_{B}$ be the B-Lift curve of $\gamma$, then we can write:

$$
\begin{gathered}
\gamma_{B}=B \quad, \quad \gamma_{B}^{\prime}=-\tau N \\
T_{B}(s)=\frac{\gamma_{B}^{\prime}}{\left\|\gamma_{B}^{\prime}\right\|}=\frac{-\tau N}{|\tau|}=-N \quad(\tau>0),
\end{gathered}
$$




$$
\begin{gathered}
\gamma_{B}^{\prime \prime}=-\tau^{\prime} N-\tau(-\kappa T+\tau B), \\
\gamma_{B}^{\prime \prime}=\kappa \tau T-\tau^{\prime} N-\tau^{2} B, \\
\gamma_{B}^{\prime} \times \gamma_{B}^{\prime \prime}=\tau^{3} T+\kappa \tau^{2} B \\
\left\|\gamma_{B}^{\prime} \times \gamma_{B}^{\prime \prime}\right\|=\tau^{2} \sqrt{\kappa^{2}+\tau^{2}}
\end{gathered}
$$

From $B_{B}(s)=\frac{\gamma_{B}^{\prime} \times \gamma_{B}^{\prime \prime}}{\left\|\gamma_{B}^{\prime} \times \gamma_{B}^{\prime \prime}\right\|}$, the equations (4) and (5) we get

$$
B_{B}(s)=\frac{\tau}{\|W\|} T+\frac{\kappa}{\|W\|} B .
$$

Using (2) and (6), we have

$$
N_{B}(s)=B_{B}(s) \times T_{B}(s)=\frac{\kappa}{\|W\|} T-\frac{\tau}{\|W\|} B .
$$

From the equations (2), (6) and (7), the proof is completed.

Theorem 6 Let $\gamma_{B}$ be the B-Lift curve of a regular curve $\gamma$ in $\mathbb{R}^{3}$. Then, we have the following formulas:

$$
\kappa_{B}(s)=\frac{\sqrt{\kappa^{2}+\tau^{2}}}{\tau} \quad, \quad \tau_{B}(s)=\frac{\kappa^{\prime} \tau-\kappa \tau^{\prime}}{\tau\left(\kappa^{2}+\tau^{2}\right)},
$$

where $\kappa_{B}$ and $\tau_{B}$ are curvature and torsion of $\gamma_{B}$, respectively.

Proof. From (5), we know

$$
\left\|\gamma_{B}^{\prime} \times \gamma_{B}^{\prime \prime}\right\|=\tau \sqrt{\kappa^{2}+\tau^{2}} \quad, \quad\left\|\gamma_{B}^{\prime}\right\|=\tau .
$$

Since $\kappa_{B}=\frac{\left\|\gamma_{B}^{\prime} \times \gamma_{B}^{\prime \prime}\right\|}{\left\|\gamma_{B}^{\prime}\right\|^{3}}$ is provided, we obtain the following equation:

$$
\kappa_{B}(s)=\frac{\sqrt{\kappa^{2}+\tau^{2}}}{\tau} .
$$

The torsion of $\gamma_{B}$ is given as

$$
\tau_{B}=\frac{<\gamma_{B}^{\prime} \times \gamma_{B}^{\prime \prime}, \gamma_{B}^{\prime \prime \prime}>}{\left\|\gamma_{B}^{\prime} \times \gamma_{B}^{\prime \prime}\right\|^{2}} .
$$

Using (3), we get

$$
\gamma_{B}^{\prime \prime \prime}=\left(\kappa^{\prime} \tau+2 \kappa \tau^{\prime}\right) T+\left(\kappa^{2} \tau-\tau^{\prime \prime}+\tau^{3}\right) N-3 \tau \tau^{\prime} B .
$$

From (4), (7) and (11), we have

$$
\tau_{B}(s)=\frac{\kappa^{\prime} \tau-\kappa \tau^{\prime}}{\tau\left(\kappa^{2}+\tau^{2}\right)} .
$$


Corollary 1 Let $\gamma_{B}$ and $\bar{\gamma}$ be the B-Lift curve and the natural lift curve of the regular curve $\gamma$, respectively. Then the following equations are provided:

$$
\begin{aligned}
T_{B}(s) & =-\bar{T}(s), \\
N_{B}(s) & =-\bar{N}(s), \\
B_{B}(s) & =\bar{B}(s),
\end{aligned}
$$

where $\left\{T_{B}(s), N_{B}(s), B_{B}(s)\right\}$ and $\{\bar{T}(s), \bar{N}(s), \bar{B}(s)\}$ are the Frenet vectors of $\gamma_{B}$ and $\bar{\gamma}$, respectively.

Corollary 2 Let $\gamma_{B}$ and $\bar{\gamma}$ be the B-Lift curve and the natural lift curve of the regular curve $\gamma$, respectively. Between the curvature and torsion of the B-lift curve and the natural lift curve there exists the following formula:

$$
\frac{\tau_{B}}{\kappa_{B}}=-\frac{\bar{\tau}}{\bar{\kappa}}
$$

Theorem $7 \gamma: I \rightarrow \mathbb{R}^{3}$ is a slant helix if and only if $\gamma_{B}$ is a general helix.

Proof. Assume that $\gamma$ is a slant helix. From Proposition 2, we have

$$
\sigma(s)=\frac{\kappa^{2}}{\left(\kappa^{2}+\tau^{2}\right)^{\frac{3}{2}}}\left(\frac{\tau}{\kappa}\right)^{\prime}(s)=\text { constant },
$$

where $\kappa$ and $\tau$ are curvature and torsion of the curve $\gamma$. We have to show if $\gamma_{B}$ is a general helix.

From Theorem 6, we can have

$$
\frac{\tau_{B}}{\kappa_{B}}=-\frac{\kappa^{2}}{\left(\kappa^{2}+\tau^{2}\right)^{\frac{3}{2}}}\left(\frac{\tau}{\kappa}\right)^{\prime}(s)=-\sigma(s)=\text { constant } .
$$

Then, $\gamma_{B}$ is a slant helix.

Conversely, let $\gamma_{B}$ be a slant helix. Then we can write

$$
\frac{\tau_{B}}{\kappa_{B}}=-\sigma(s)=\text { constant }
$$

Since $\sigma(\mathrm{s})=$ constant, the curve $\gamma$ is a slant helix.

Corollary $3 \gamma: I \rightarrow \mathbb{R}^{3}$ is a Darboux helix if and only if $\gamma_{B}$ is a general helix.

Theorem $8 \gamma: I \rightarrow \mathbb{R}^{3}$ is a general helix if and only if $\gamma_{B}$ is a plane curve.

Proof. Let $\gamma$ be a general helix. Then the ratio of $\frac{\tau}{\kappa}$ is constant. Hence $\left(\frac{\tau}{\kappa}\right)^{\prime}=0$ and consequently we can write

$$
-\frac{\kappa^{2}}{\left(\kappa^{2}+\tau^{2}\right)^{\frac{3}{2}}}\left(\frac{\tau}{\kappa}\right)^{\prime}(s)=\frac{\tau_{B}}{\kappa_{B}}=0
$$


Since $\frac{\tau_{B}}{\kappa_{B}}=0$,we have $\tau_{B}=0$. So, the curve $\gamma_{B}$ be a plane curve. Conversely, let $\gamma_{B}$ is a plane curve. Then, $\tau_{B}=0$ and from (14)

$$
\left(\frac{\tau}{\kappa}\right)^{\prime}(s)=0
$$

So, $\frac{\tau}{\kappa}$ is constant. Since $\frac{\tau}{\kappa}$ is constant, $\gamma$ is a general helix.

Example 1 Assume that the unit speed slant helix is given as

$$
\gamma(s)=\left(\frac{1}{6} \sin 2 s+\frac{2}{3} \sin s, \frac{1}{6} \cos 2 s+\frac{2}{3} \cos s, \frac{4 \sqrt{2}}{3} \cos \frac{s}{2}\right) .
$$

Then the Frenet vectors of the curve $\gamma$ are given as follows:

$$
\begin{aligned}
& T(s)=\left(\frac{1}{3} \cos 2 s+\frac{2}{3} \cos s,-\frac{1}{3} \sin 2 s-\frac{2}{3} \sin s,-\frac{2 \sqrt{2}}{3} \sin \frac{s}{2}\right), \\
& N(s)=\left(-\frac{4}{3 \sqrt{2}} \sin \frac{3 s}{2},-\frac{4}{3 \sqrt{2}} \cos \frac{3 s}{2},-\frac{1}{3}\right), \\
& B(s)=\left(-\frac{1}{3} \sin 2 s+\frac{2}{3} \sin s,-\frac{1}{3} \cos 2 s+\frac{2}{3} \cos s,-\frac{4}{3 \sqrt{2}} \cos \frac{s}{2}\right) .
\end{aligned}
$$

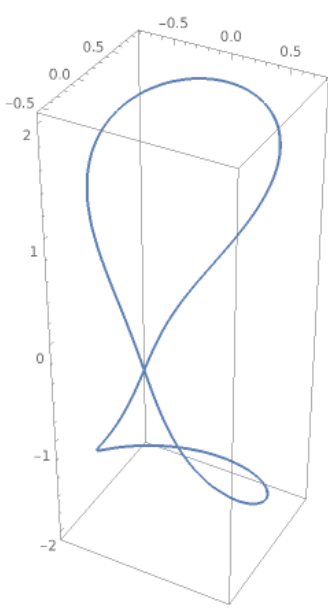

Fig. 1 The slant helix $\gamma(s)$

since $\gamma_{B}(\mathrm{~s})=\mathrm{B}(\mathrm{s})$, we have the following equations:

$$
\begin{aligned}
\gamma_{B}^{\prime}(s) \times \gamma_{B}^{\prime \prime}(s) & =\left(\frac{2 \sqrt{2}}{3} \sin ^{2} \frac{s}{2} \sin \frac{3 s}{2}, \frac{2 \sqrt{2}}{3} \sin ^{2} \frac{s}{2} \cos \frac{3 s}{2},-\frac{8}{3} \sin ^{2} \frac{s}{2}\right), \\
\kappa_{B}(s) & =\frac{\left\|\gamma_{B}^{\prime} \times \gamma_{B}^{\prime \prime}\right\|}{\left\|\gamma_{B}^{\prime}\right\|^{3}}=\frac{1}{\sin \frac{s}{2}}, \\
\tau_{B}(s) & =\frac{\left\langle\gamma_{B}^{\prime} \times \gamma_{B}^{\prime \prime}, \gamma_{B}^{\prime \prime \prime}>\right.}{\left\|\gamma_{B}^{\prime} \times \gamma_{B}^{\prime \prime}\right\|^{2}}=-\frac{1}{2 \sqrt{2}} \frac{1}{\sin \frac{s}{2}} .
\end{aligned}
$$


Therefore, we obtain

$$
\frac{\tau_{B}}{\kappa_{B}}=-\frac{1}{2 \sqrt{2}}=\text { constant }
$$

Since the ratio of the curvatures are constant, the curve $\gamma_{B}$ is a general helix.

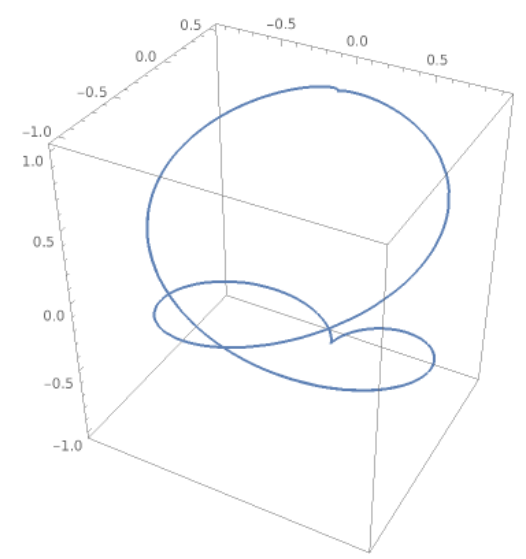

Fig. 2 The general helix $\gamma_{B}(s)$

Example 2 Consider the unit speed circular helix with

$$
\gamma(s)=\left(\cos \frac{s}{\sqrt{2}}, \sin \frac{s}{\sqrt{2}}, \frac{s}{\sqrt{2}}\right) .
$$

After some calculations, the Frenet operators of the curve $\gamma$

$$
\begin{aligned}
& T(s)=\left(-\frac{1}{\sqrt{2}} \sin \frac{s}{\sqrt{2}}, \frac{1}{\sqrt{2}} \cos \frac{s}{\sqrt{2}}, \frac{1}{\sqrt{2}}\right), \\
& N(s)=\left(-\cos \frac{s}{\sqrt{2}},-\sin \frac{s}{\sqrt{2}}, 0\right), \\
& B(s)=\left(\frac{1}{\sqrt{2}} \sin \frac{s}{\sqrt{2}},-\frac{1}{\sqrt{2}} \cos \frac{s}{\sqrt{2}}, \frac{1}{\sqrt{2}}\right) .
\end{aligned}
$$

From (1), we have the following equations:

$$
\begin{aligned}
\gamma_{B}^{\prime}(s) & =\left(\frac{1}{2} \cos \frac{s}{\sqrt{2}}, \frac{1}{2} \sin \frac{s}{\sqrt{2}}, 0\right), \\
\gamma_{B}^{\prime \prime}(s) & =\left(-\frac{1}{2 \sqrt{2}} \sin \frac{s}{\sqrt{2}}, \frac{1}{2 \sqrt{2}} \cos \frac{s}{\sqrt{2}}, 0\right), \\
\gamma_{B}^{\prime}(s) \times \gamma_{B}^{\prime \prime}(s) & =\left(0,0, \frac{1}{4 \sqrt{2}}\right), \\
\gamma_{B}^{\prime \prime}(s) & =\left(-\frac{1}{4} \cos \frac{s}{\sqrt{2}},-\frac{1}{4} \sin \frac{s}{\sqrt{2}}, 0\right) .
\end{aligned}
$$




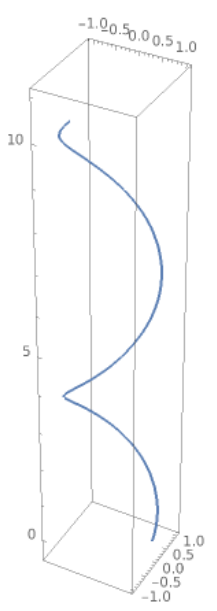

Fig. 3 The circular helix $\gamma(s)$

If the above equations and $(10)$ are used, $\tau_{B}(\mathrm{~s})=0$ is obtained. Then the curve $\gamma_{B}(s)$ is a plane curve.

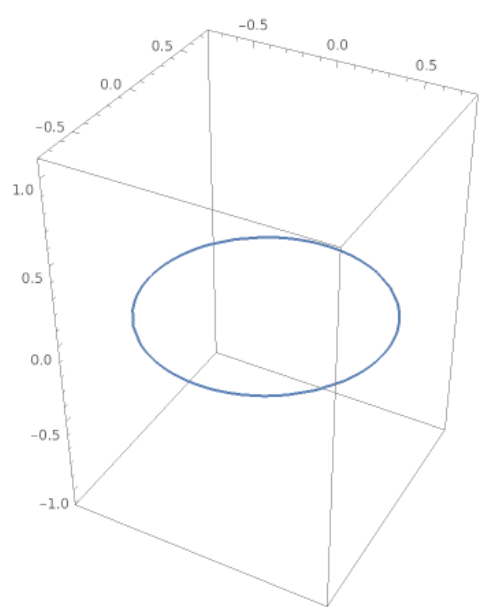

Fig. 4 The plane curve $\gamma_{B}(s)$

\section{Conclusion}

In this article, introduced the B-lift curve and the relation between Frenet operators of the natural lift curve and the B-lift curve of the main curve are 
given. Based on the definition of B-lift, the situation of the curve being slant helix, darboux helix and general helix is examined.

\section{Conflict of interest}

The authors declare that they have no conflict of interest.

\section{References}

1. M. Do. Carmo, Differential Geometry of Curves and Surfaces PrenticeHall,Inc.,Englewood Cliffs, New Jersey, (1976).

2. B. O'Neill, Elementary Differential Geometry, Academic Press New York and London, (1966).

3. J.A. Thorpe, Elementary Topics in Differential Geometry, Springer Verlag, New York, Heidelberg-Berlin, (1979).

4. E. Ergün, M. Çalı̧kan, On natural lift of a curve, Pure Mathematical Sciences, Vol. 1, 81-85 (2012).

5. E. Ergün, M. Bilici, M. Çalışkan, The Frenet vector fields and the curvatures of the natural lift curve, The Bulletin of Soc. for Mathematical Services and Standards 2 , 38-43 (2012).

6. M.A.Lancret, Mémoire sur les courbes à double courbure, Mémoires présentés à l'Institut 1, 416-454 (1806) .

7. P. D. Scofield, Curves of constant precession, The American Mathematical Monthly 102, 531-537 (1995).

8. M. Barros, General helices and a theorem of Lancret, Proc. Amer. Math. Soc. 125, no.5, 1503-1509 (1997).

9. S. Izumiya and N. Takeuchi, New special curves and developable surfaces, Turk J. Math., 28, 153-163 (2004)

10. L. Kula, Y. Yayli, On slant helix and its spherical indicatrix, Applied Mathematics and Computation 169, 600-607 (2005).

11. L. Kula, N. Ekmekci, Y. Yayl, K. İlarslan, Characterizations of slant helices in Euclidean 3-space, Turk J Math 34, 261-273 (2010).

12. E. Zıplar, A. Şenol, Y. Yayl, On Darboux Helices in Euclidean 3-Space, Global Journals Inc., Vol. 12, 73-80 (2012).

13. E. Karaca, M. Çalışkan, Ruled Surfaces and Tangent Bundle of Unit 2-Sphere of Natural Lift Curves, GU J Sci, 33(3), 751-759 (2020). 